PROCEEDINGS OF THE

AMERICAN MATHEMATICAL SOCIETY

Volume 136, Number 12, December 2008, Pages 4427-4436

S 0002-9939(08)09451-3

Article electronically published on June 20, 2008

\title{
SPECTRAL ANALYSIS OF BROWNIAN MOTION WITH JUMP BOUNDARY
}

\author{
YUK J. LEUNG, WENBO V. LI, AND RAKESH
}

(Communicated by Richard C. Bradley)

\begin{abstract}
Consider a family of probability measures, indexed by $\partial D$, on a bounded open region $D \subset \mathbb{R}^{d}$ with a smooth boundary. For any starting point inside $D$, we run a standard $d$-dimensional Brownian motion in $\mathbb{R}^{d}$ until it first exits $D$, at which time it jumps to a point inside the domain $D$ according to the jump measure at the exit point and starts a new Brownian motion. The same evolution is repeated independently each time the process reaches the boundary. We study the exponential rate at which the transition distribution of the process converges to its invariant measure, in terms of the spectral gap of the generator. In particular, we prove two conjectures of I. Ben-Ari and R. Pinsky for an interval (see J. Funct. Anal. 251 (2007), 122-140, and preprint (2007)) by studying when a combination of the sine and cosine transforms of probability measures on an interval has only real zeros.
\end{abstract}

\section{INTRODUCTION}

Let $D$ be a bounded, open, connected subset of $\mathbb{R}^{d}$ with a $C^{2}$-smooth boundary and let $\left\{\nu_{y}: y \in \partial D\right\}$ be a family of probability measures on $D$. For any starting point $x \in D$, we run a standard $d$-dimensional Brownian motion $B(t) \in \mathbb{R}^{d}$ until it first exits $D$ at time $\tau$, at which time it jumps to a point in the domain $D$ according to the measure $\nu_{B(\tau)}$ at the exit point and starts the Brownian motion afresh. This evolution is repeated independently each time the process reaches the boundary, and the resulting diffusion process will be called Brownian motion with jump boundary (BMJ). BMJ models have been used for a variant of the Fleming-Viot branching process in 4, Brownian flow on a finite interval with jump boundary conditions in [10, and the behavior of the double knock-out barrier options in the study of financial derivatives in $[\underline{5}, 6,6$. 7 .

If, for a fixed $p \in D, \nu_{y}=\delta_{p}$ for all $y \in \partial D$, i.e. the jumps are deterministic and concentrated at a single point $p \in D$, then the ergodicity of BMJ was studied in [5], 8 using Laplace transform methods and the theory of analytic semigroups. In the case where $\nu_{y}=\nu$ for all $y \in \partial D$ ( $\nu$ need not be a point measure), the ergodicity of BMJ (as an important special case of elliptic operators) was systematically studied in 2 using a functional analytic approach. Recently, the most general case, where $\nu_{y}$ depends continuously on its exit point $y \in \partial D$, was studied in [3]. The key results in [2], 3] address the construction of the invariant probability measure and

Received by the editors September 25, 2007, and, in revised form, November 13, 2007.

2000 Mathematics Subject Classification. Primary 60J25; Secondary 30D10, 47D07, 60J35.

The second author was supported in part by NSF Grant DMS-0505805.

(C)2008 American Mathematical Society Reverts to public domain 28 years from publication 
relate the rate of convergence of the transition distribution to the invariant measure to the spectral gap of the generator of BMJ.

Consider the Markov process $X(t)$ with $\nu_{y}$ depending continuously on its exit point $y \in \partial D$. If $p(t, x, \cdot)$ represents the transition probability measure for this Markov process, then it was shown in 3 that $p(t, x, \cdot)$ approaches a unique stationary invariant measure $\mu$ on $D$ with the density

$$
\mu(d y)=\frac{\int_{D} G^{D}(x, y) d \nu(x) d y}{\int_{D} \int_{D} G^{D}(x, y) d \nu(x) d y}=\frac{G^{D}(\nu, y) d y}{\int_{D} G^{D}(\nu, y) d y},
$$

where $\nu=\int_{\partial D} \nu_{y} d m(y)$ and $m$ is the unique invariant measure on $\partial D$ such that

$$
m(y)=\int_{\partial D} \int_{D} H(z, y) d \nu_{x}(z) d m(x)=\int_{\partial D} H\left(\nu_{x}, y\right) d m(x) .
$$

Here $H(x, y)$, for every $(x, y) \in D \times \partial D$, is the density of the harmonic measure associated with the first exit location, and the Dirichlet Green's function $G^{D}(x, y)$ is the solution of

$$
\begin{gathered}
\frac{1}{2} \Delta_{x} G^{D}(x, y)=-\delta(x-y), \quad x \in D, \\
G^{D}(x, y)=0, \quad x \in \partial D .
\end{gathered}
$$

Equivalently, $G^{D}(x, y)=\int_{0}^{\infty} p^{D}(t, x, y) d t$ is the 0-potential of the transition subprobability function $p^{D}(t, x, y)$ of the absorbed Brownian motion on $D$.

The article [3] also describes the rate of convergence of $p(t, x, \cdot)$ to the stationary measure. Consider the eigenvalues of the non-local eigenvalue problem

$$
\frac{1}{2} \Delta u=\lambda u \quad \text { on } D, \quad u(y)=\int_{D} u(x) d \nu_{y}(x), \quad y \in \partial D .
$$

In 3] it was shown that the eigenvalues of (1.4) are countable and do not have an accumulation point, the non-zero eigenvalues have a negative real part, and

$$
\lim _{t \rightarrow \infty} \frac{1}{t} \sup _{f \in L^{\infty}(D),\|f\|_{\infty} \leq 1} \log \left\|\mathbb{E}_{x} f(X(t))-\int_{D} f d \mu\right\|_{\infty}=\gamma_{1},
$$

where

$$
\gamma_{1}=\sup \{\Re \lambda: 0 \neq \lambda \text { is an eigenvalue of (1.4) }\}
$$

is the spectral gap.

Throughout the article, the superscript $D$ will correspond to the Dirichlet boundary condition $u=0$ on $\partial D$. For any probability measure $\nu$ on $D$ and any function $g$ on $D$ we will use the notation

$$
g(\nu):=\int_{D} g(x) d \nu(x), \quad \mathbb{E}_{\nu} g:=\int_{D} \mathbb{E}_{x} g d \nu(x) .
$$

Our first two results are about the BMJ on an interval $D=(a, b)$ with jump measures $\nu_{a}$ and $\nu_{b}$ on $D$. In an earlier version of this article, independent of [3], we identified the invariant measure $\mu$, in very explicit form, for the $D=(a, b)$ case. We give the instructive proof of this result. This explicit form may also be derived from (1.1) once the invariant measure $m$ on the boundary and the associated harmonic density $H(x, y)$ are identified. 
Proposition 1.1. Let $X(t)$ be the BMJ process on $(a, b)$ associated to the probability measures $\nu_{a}, \nu_{b}$ and let $m_{a}, m_{b}$ be the means of $\nu_{a}, \nu_{b}$. Then $X(t)$ has a unique invariant measure $\mu$ given by

$$
\mu(d y)=\frac{\left(b-m_{b}\right) G^{D}\left(\nu_{a}, y\right) d y+\left(m_{a}-a\right) G^{D}\left(\nu_{b}, y\right) d y}{\left(b-m_{b}\right) \int_{a}^{b} G^{D}\left(\nu_{a}, y\right) d y+\left(m_{a}-a\right) \int_{a}^{b} G^{D}\left(\nu_{b}, y\right) d y},
$$

where

$$
G^{D}(x, y)=\frac{2}{b-a}(b-\max (x, y))(\min (x, y)-a), \quad x, y \in(a, b),
$$

is the solution of (1.2), (1.3) for $D=(a, b)$.

Corresponding to (1.4) for $D=(a, b)$, consider the eigenvalues of the non-local eigenvalue problem:

$$
\begin{gathered}
\frac{1}{2} u^{\prime \prime}=\lambda u \quad \text { on }(a, b), \\
u(a)=\int_{a}^{b} u(x) d \nu_{a}(x), \quad u(b)=\int_{a}^{b} u(x) d \nu_{b}(x) .
\end{gathered}
$$

Define the spectral gap

$$
\gamma_{1}\left(\nu_{a}, \nu_{b}\right)=\sup \{\Re \lambda: 0 \neq \lambda \text { is an eigenvalue of (1.7), (1.8) }\} .
$$

We have the following results.

Theorem 1.2. If $\nu_{a}, \nu_{b}$ are probability measures on $(a, b)$, then all the eigenvalues of (1.7), (1.8) are real and non-positive. As a consequence

$$
\sup _{\nu_{a}, \nu_{b}} \gamma_{1}\left(\nu_{a}, \nu_{b}\right)=\lambda_{0}^{D}=-\frac{\pi^{2}}{2(b-a)^{2}},
$$

and if $\nu_{a}=\nu_{b}=\nu$, then

$$
\gamma_{1}(\nu, \nu)=\lambda_{1}^{D}=-\frac{2 \pi^{2}}{(b-a)^{2}}
$$

Here $\lambda_{0}^{D}, \lambda_{1}^{D}$ are the largest and the second largest Dirichlet eigenvalues for $\Delta / 2$ on $(a, b)$.

In (1.9) the supremum is never attained, as shown in the proof of Theorem 1.2 and in (1.10) the RHS is independent of $\nu$, which is quite surprising and we do not have a good probabilistic explanation for it. The equations (1.9) and (1.10) were conjectured in [3] and 22 respectively, and Proposition 2 in [2] shows that (1.10) is a consequence of the main result in Theorem 1.2. In [3, it was also conjectured that

$$
\inf _{\nu_{a}, \nu_{b}} \gamma_{1}\left(\nu_{a}, \nu_{b}\right)=\lambda_{2}^{D}=-\frac{9 \pi^{2}}{2(b-a)^{2}},
$$

where $\lambda_{2}^{D}$ is the third largest Dirichlet eigenvalue; in [3] it was shown that the value on the right-hand side of (1.11) is attained at $\nu_{a}=\delta_{(a+2 b) / 3}$ and $\nu_{b}=\delta_{(2 a+b) / 3}$. This conjecture is still open and is related to our convexity conjecture that for all $h \in[0,1]$

$$
h \gamma_{1}\left(\nu_{a}, \nu_{b}\right)+(1-h) \gamma_{1}\left(\nu_{a}^{\prime}, \nu_{b}^{\prime}\right) \leq \gamma_{1}\left(h \nu_{a}+(1-h) \nu_{a}^{\prime}, h \nu_{a}+(1-h) \nu_{b}^{\prime}\right) .
$$


Our third result concerns BMJ on the unit ball in $\mathbb{R}^{d}$ with the assumption that $\nu_{y}$ is independent of $y \in \partial D$. Let $B$ denote the open unit ball in $\mathbb{R}^{d}$ and $\nu$ a probability measure on $B$. Then (1.4) takes the form of the eigenvalue problem

$$
\frac{1}{2} \Delta u=\lambda u \quad \text { in } B,\left.\quad u\right|_{\partial B}=\int_{B} u d \nu .
$$

If the non-zero eigenvalue of (1.12) with the largest real part is real, then there is a useful upperbound $\gamma_{1}(\nu)<\lambda_{0}^{D}$ as stated in Theorem 5 of [2]. This motivates the study of the question of when the eigenvalues of (1.12) are real.

Theorem 1.3. Suppose $d>1$ is odd and $\nu$ is an absolutely continuous (w.r.t. Lebesgue measure) probability measure on the open unit ball $B$ in $\mathbb{R}^{d}$ with a density in $L^{2}(B)$. If $r^{-d} \nu\left(\left\{x \in \mathbb{R}^{d}:|x|<r\right\}\right)$ is an increasing function of $r$ on $(0,1)$, then the eigenvalues of (1.12) are real (and non-positive).

The odd $d$ case reduces to the study of the zeros of the Fourier transform of measures; we have no results for the even $d$ case because we do not have tools to study the zeros of the Fourier-Bessel transforms of measures.

Remark 1.4. For general $\nu$, even in the $D=B$ case, (1.12) may have complex eigenvalues as seen when $d=3, \nu=\delta_{p}$ with $|p|=1 / 4$ (see section 4), but we do not have any examples for which the non-zero eigenvalue with the largest real part is not real.

The remaining sections are organized as follows. In section 2 we prove Proposition 1.1, in section 3 we prove Theorem 1.2 and in section 4 we prove Theorem 1.3 ,

\section{Proof of Proposition 1.1}

We give the proof when $a=0$ and $b=1$; the $(a, b)$ case is similar. We relabel $\nu_{a}$ as $\nu_{0}$ and $\nu_{b}$ as $\nu_{1}$.

Green's function $G^{D}(x, y)$ for (1.2), (1.3) should be considered as one-half the potential at the point $x$ due to a unit positive electrical charge placed at $y$ inside the grounded boundary $\partial D$. From the probabilistic point of view,

$$
\begin{aligned}
G^{D}(x, y) d y & =\mathbb{E}_{x} \int_{0}^{\tau} \mathbb{I}_{\{B(t) \in d y\}} d t \\
& =\int_{0}^{\infty} \mathbb{P}_{x}(B(t) \in d y, \tau>t) d t=\int_{0}^{\infty} p^{D}(t, x, y) d t d y
\end{aligned}
$$

is the expected time that the standard Brownian motion path starting at $x$ spends in $d y$ before hitting $\partial D$. Another way of writing this is that

$$
\int_{D} f(y) G^{D}(x, y) d y=\mathbb{E}_{x} \int_{0}^{\tau} f(B(t)) d t, \quad f \in L^{\infty} .
$$

Consider the semigroup $\left\{T_{t}\right\}_{t \geq 0}$ from $L^{\infty}$ to $L^{\infty}$ associated with BMJ $X$, i.e.

$$
T_{t} f(x)=\mathbb{E}_{x} f(X(t))=\int_{0}^{1} p(t, x, y) f(y) d y, \quad f \in L^{\infty} .
$$


We prove that $\mu$ is an invariant measure for $\left\{T_{t}\right\}_{t \geq 0}$. Since $X$ coincides with the absorbed Brownian motion up to the stopping time $\tau$, we have

$$
\begin{aligned}
\int_{0}^{1}\left(T_{t} f\right)(y) & G^{D}\left(\nu_{0}, y\right) d y=\int_{0}^{1} \int_{0}^{1}\left(T_{t} f\right)(y) G^{D}(x, y) d y d \nu_{0}(x) \\
& =\int_{0}^{1} \mathbb{E}_{x} \int_{0}^{\tau}\left(T_{t} f\right)(X(s)) d s d \nu_{0}(x)=\mathbb{E}_{\nu_{0}} \int_{0}^{\tau} \mathbb{E}_{X(s)} f(X(t)) d s \\
& =\mathbb{E}_{\nu_{0}} \int_{0}^{\tau} f(X(s+t)) d s=\mathbb{E}_{\nu_{0}} \int_{t}^{t+\tau} f(X(s)) d s \\
& =\mathbb{E}_{\nu_{0}} \int_{0}^{\tau} f(X(s)) d s+\mathbb{E}_{\nu_{0}} \int_{\tau}^{t+\tau} f(X(s)) d s-\mathbb{E}_{\nu_{0}} \int_{0}^{t} f(X(s)) d s .
\end{aligned}
$$

For the middle term in the last line, we have with the process starting at $x$,

$$
\begin{aligned}
\mathbb{E}_{x} \int_{\tau}^{t+\tau} f(X(s)) d s=\mathbb{E}_{x} & \left(\int_{\tau}^{t+\tau} f(X(s)) d s \mid X(\tau)=0\right) \cdot \mathbb{P}_{x}(X(\tau)=0) \\
& +\mathbb{E}_{x}\left(\int_{\tau}^{t+\tau} f(X(s)) d s \mid X(\tau)=1\right) \cdot \mathbb{P}_{x}(X(\tau)=1) \\
= & \mathbb{E}_{\nu_{0}} \int_{0}^{t} f(X(s)) d s \cdot(1-x)+\mathbb{E}_{\nu_{1}} \int_{0}^{t} f(X(s)) d s \cdot x,
\end{aligned}
$$

where $\mathbb{P}_{x}(X(\tau)=0)=\mathbb{P}_{x}(B(\tau)=0)=1-x$ and $\mathbb{P}_{x}(X(\tau)=1)=x$ follows from the well-known one-dimensional hitting probability associated with continuous gambler's ruin probability.

Substituting (2.3) into (2.2), we obtain

$$
\begin{aligned}
\int_{0}^{1}\left(T_{t} f\right)(y) G^{D}\left(\nu_{0}, y\right) d y= & \int_{0}^{1} f(y) G^{D}\left(\nu_{0}, y\right) d y \\
& +m_{0}\left(\mathbb{E}_{\nu_{1}} \int_{0}^{t} f(X(s)) d s-\mathbb{E}_{\nu_{0}} \int_{0}^{t} f(X(s)) d s\right) .
\end{aligned}
$$

Similarly, we find

$$
\begin{aligned}
\int_{0}^{1}\left(T_{t} f\right)(y) G^{D}\left(\nu_{1}, y\right) d y= & \int_{0}^{1} f(y) G^{D}\left(\nu_{1}, y\right) d y \\
& +\left(1-m_{1}\right)\left(\mathbb{E}_{\nu_{0}} \int_{0}^{t} f(X(s)) d s-\mathbb{E}_{\nu_{1}} \int_{0}^{t} f(X(s)) d s\right) .
\end{aligned}
$$

Thus for the "mixed" Green's function

$$
G(y)=\left(1-m_{1}\right) G^{D}\left(\nu_{0}, y\right)+m_{0} G^{D}\left(\nu_{1}, y\right),
$$

we have $\int_{0}^{1}\left(T_{t} f\right)(y) G(y) d y=\int_{0}^{1} f(y) G(y) d y$, which implies $\mu(y)=G(y) / \int_{0}^{1} G(y) d y$ is the invariant measure in (1.6).

\section{Proof of Theorem 1.2}

For a simpler presentation, we prove Theorem 1.2 when $(a, b)=(0,1)$ and we relabel the jump measures as $\nu_{0}$ and $\nu_{1}$; the general case is similar. We replace $\lambda$ by $-z^{2} / 2$ for convenience, so $\lambda$ will be real and non-positive if $z$ is real, which is what we intend to prove. The general solution of (1.7) is

$$
u(t)=A \cos (z t)+B \sin (z t),
$$


so the boundary conditions (1.8) force

$$
\begin{aligned}
A & =\int_{0}^{1}(A \cos (z s)+B \sin (z s)) d \nu_{0}(s), \\
A \cos (z)+B \sin (z) & =\int_{0}^{1}(A \cos (z t)+B \sin (z t)) d \nu_{1}(t) .
\end{aligned}
$$

This system in $A, B$ will have a non-zero solution if and only if $F(z)=0$, where

$$
\begin{aligned}
F(z):=\sin z & -\int_{0}^{1} \sin (z t) d \nu_{1}(t)-\int_{0}^{1} \sin (z(1-s)) d \nu_{0}(s) \\
& +\int_{0}^{1} \int_{0}^{1} \sin (z(t-s)) d \nu_{0}(s) d \nu_{1}(t) .
\end{aligned}
$$

We show that the equation $F(z)=0$ has only real solutions. Note that $F(z)$ has an infinite number of zeros because of the Hadamard Factorization Theorem (see page 211 of [1] ) and the fact that $F(z)$ is odd and the entire function $F(\sqrt{z}) / \sqrt{z}$ is of order $1 / 2$.

The starting point is an argument used by Pólya in [12 (also see [13]) to prove that the finite sine and cosine transforms of increasing functions have only real zeros. For any positive integer $n$, we approximate these integrals using a subdivision of $(0,1)$ into $n$ parts. Define

$$
\begin{aligned}
& a_{n}=\nu_{0}\left(\left(\frac{n-1}{n}, 1\right)\right), a_{j}=\nu_{0}\left(\left(\frac{j-1}{n}, \frac{j}{n}\right]\right), j=1, \cdots, n-1, \\
& b_{0}=\nu_{1}\left(\left(0, \frac{1}{n}\right)\right), b_{k}=\nu_{1}\left(\left[\frac{k}{n}, \frac{k+1}{n}\right)\right), k=1, \cdots, n-1,
\end{aligned}
$$

and

$$
F_{n}(z):=\sin (z)-\sum_{k=0}^{n-1} b_{k} \sin \left(\frac{k z}{n}\right)-\sum_{j=1}^{n} a_{j} \sin \left(\frac{z(n-j)}{n}\right)+\sum_{k=0}^{n-1} \sum_{j=1}^{n} a_{j} b_{k} \sin \left(\frac{z(k-j)}{n}\right) .
$$

Then the analytic function $F_{n}(z)$ converges to the analytic function $F(z)$ uniformly on compact subsets. Hence, by Hurwitz's theorem (see page 178 of [1]), $F(z)$ will have only real zeros if we can show that $F_{n}(z)$ has only real zeros. Define

$$
G_{n}(w):=w^{n}-\sum_{k=0}^{n-1} b_{k} w^{k}-\sum_{j=1}^{n} a_{j} w^{n-j}+\sum_{k=0}^{n-1} \sum_{j=1}^{n} a_{j} b_{k} w^{k-j}
$$

Then $F_{n}(z)=\frac{1}{2 i}\left(G_{n}(w)-G_{n}\left(w^{-1}\right)\right)$ if we take $w=e^{i z / n}$, and $F_{n}(z)$ has only real zeros iff all the zeros of $G_{n}(w)-G_{n}\left(w^{-1}\right)$ are on the unit circle. We show that $G_{n}(w)-G_{n}\left(w^{-1}\right)$ has no zeros inside the unit circle, so it will not have any zeros outside the unit circle too, and we will be done.

Now $G_{n}(w)=w^{-n} P_{n}(w) Q_{n}(w)$ where

$$
P_{n}(w):=w^{n}-\sum_{k=0}^{n-1} b_{k} w^{k}, \quad Q_{n}(w):=w^{n}-\sum_{j=1}^{n} a_{j} w^{n-j} .
$$

We claim that the zeros of $P_{n}(w)$ and $Q_{n}(w)$ are in the closed unit disk and, for sufficiently large $n$, at least one zero, either of $P_{n}(w)$ or of $Q_{n}(w)$, is in the open 
unit disk. If $w_{0}$ is a zero of $P_{n}(w)$ with $\left|w_{0}\right| \geq 1$, then $w_{0}^{n}=\sum_{k=0}^{n-1} b_{k} w_{0}^{k}$, so

$$
1=\left|\sum_{k=0}^{n-1} b_{k} w_{0}^{k-n}\right| \leq \sum_{k=0}^{n-1} b_{k}\left|w_{0}\right|^{k-n} \leq \sum_{k=0}^{n-1} b_{k}=1 .
$$

So $\left|w_{0}\right|=1$; a similar argument applies to $Q_{n}(w)$. Now if all the zeros of $P_{n}(w)$ or $Q_{n}(w)$ were on the unit circle, then their products would have absolute value 1 , which would imply $b_{0}=1$ or $a_{n}=1$. If this were true for an infinite number of $n$, then letting $n$ approach infinity we would have $\nu_{0}(\emptyset)=1$ or $\nu_{1}(\emptyset)=1$, which is not true.

Suppose $P_{n}(w)=\prod_{j=1}^{n}\left(w-p_{j}\right)$ and $Q_{n}(w)=\prod_{j=1}^{n}\left(w-q_{j}\right)$. Note that the $p_{j}$ and $q_{j}$ are either real or occur in complex conjugate pairs because the coefficients of $P_{n}(w)$ and $Q_{n}(w)$ are real. The zeros of $G_{n}(w)-G_{n}\left(w^{-1}\right)$ are the solutions of

$$
1=\frac{G_{n}(w)}{G_{n}\left(w^{-1}\right)}=\frac{\prod_{j=1}^{n}\left(w-p_{j}\right)\left(w-q_{j}\right)}{\prod_{j=1}^{n}\left(1-p_{j} w\right)\left(1-q_{j} w\right)}=\prod_{j=1}^{n} \frac{w-p_{j}}{1-\overline{p_{j}} w} \prod_{j=1}^{n} \frac{w-q_{j}}{1-\overline{q_{j}} w} .
$$

For any $\alpha$ with $|\alpha|<1$, the Mobius transformation $(w-\alpha) /(1-\bar{\alpha} w)$ maps the open unit disk to the open unit disk; if $|\alpha|=1$, then all the points are mapped to the unit circle. Since one of the $p_{j}$ and one of the $q_{j}$ are in the open unit disk, it is clear that (3.5) cannot hold for any $w$ in the open unit disk.

Next we show how (1.9) follows from the fact that the eigenvalues of (1.7), (1.8) are real. From (3.4), $\lambda=-z^{2} / 2$ will be an eigenvalue of (1.7), (1.8) iff

$$
F(z):=\int_{0}^{1} \int_{0}^{1} \kappa(s, t ; z) d \nu_{0}(s) d \nu_{1}(t)=0
$$

where

$$
\begin{aligned}
\kappa(s, t ; z) & :=(1-\cos (z s))(\sin (z)-\sin (z t))+\sin (z s)(\cos (z)-\cos (z t)) \\
& =\sin \left(\frac{t-s+1}{2} z\right) \sin \left(\frac{t-1}{2} z\right) \sin \left(\frac{s}{2} z\right) .
\end{aligned}
$$

Since $\kappa(s, t, z)>0$ for all $z \in(-\pi, 0)$ and for all $(s, t) \in(0,1) \times(0,1)$, (1.7), (1.8) will have no eigenvalues in $\left[-\pi^{2} / 2,0\right)$. Further, if we take $\nu_{0}=\delta_{p}$ and $\nu_{1}=\delta_{q}$ for $p, q$ in $(0,1)$, then, as stated in Proposition 2 of $[3]$,

$$
\gamma_{1}\left(\nu_{0}, \nu_{1}\right)=-\frac{2 \pi^{2}}{\max \left(p^{2},(1-q)^{2},(1-p+q)^{2}\right)},
$$

which approaches $-\pi^{2} / 2$ as $(p, q)$ approach $(0,1)$. This proves (1.9).

\section{Proof of Theorem 1.3}

The densely defined operator $\phi \mapsto \Delta \phi$ is not a symmetric operator on $L^{2}(\bar{B})$ for $\phi$ restricted to functions in $C^{\infty}(\bar{B})$ satisfying the boundary condition in (1.12); hence the theory for self-adjoint operators is not applicable in any obvious way to prove that the eigenvalues are real.

Below $S$ denotes the boundary of $B$. Let $\lambda$ be an eigenvalue and $u$ the corresponding eigenfunction for (1.12). We assume that $u \in H^{s}(B)$ for some $s>1 / 2$ (so $u$ has traces on $S$ ). Since $u \in L^{2}(B)$ and $\nu$ has an $L^{2}$ density, it is clear that $u$ will be integrable w.r.t. $\nu$ so that the boundary condition in (1.12) makes sense. Since $u$ is constant on $S$ because of the boundary condition in (1.12) and the RHS 
of (1.12) is in $H^{s}(B)$, using Theorem 4.18 in [11] repeatedly, one may conclude that $u$ has continuous derivatives of all orders on $\bar{B}$.

Theorem 2 in 2 characterizes the eigenvalues of (1.12), but for the special domain $B$ and when $\mu_{r e v}$ is the Lebesgue measure, we have more specific information. As in [2, let $\left\{\lambda_{n}^{D}\right\}_{n=0}^{\infty}$ be the Dirichlet eigenvalues of $\Delta / 2$ and $\left\{\phi_{n}^{D}\right\}_{n=0}^{\infty}$ the corresponding eigenfunctions with unit $L^{2}$ norm; the $\lambda_{n}^{D}$ are known to be real. From equation (3.5) in the proof of Theorem 2 in [2], either $\lambda$ is one of the Dirichlet eigenvalues or (our $u$ is the $v$ in [2])

$$
u=c+\lambda c \sum_{n=0}^{\infty} \frac{F_{n}}{\lambda_{n}^{D}-\lambda} \phi_{n}^{D}
$$

for some constant $c$, where $F_{n} \equiv \int_{B} \phi_{n}^{D}(x) d x$. The Dirchlet eigenfunctions $\phi_{n}^{D}(x)=$ $a_{n}(r) b_{n}(\theta)$, where $r=|x|$ and $\theta=x /|x|$, for some homogeneous harmonic polynomial $b_{n}(x)$. From the properties of homogeneous harmonic polynomials (see [14]) either $b_{n}(x)=$ constant or $\int_{|\theta|=1} b_{n}(\theta) d \theta=0$. Hence if $\phi_{n}^{D}$ is not a spherically symmetric function, then $F_{n}=\int_{B} \phi_{n}^{D}(x) d x=0$, implying that $u$, as given by (4.1), is spherically symmetric. Hence either $\lambda$ is one of the Dirichlet eigenvalues (and real) or the eigenfunction $u$ is spherically symmetric. We now examine the spherically symmetric eigenfunctions of (1.12).

If $\lambda=-z^{2} / 2$ is an eigenvalue corresponding to a smooth spherically symmetric eigenfunction $a(r)$, then $a(r)$ is a solution of the boundary value problem

$$
\begin{gathered}
a_{r r}+\frac{d-1}{r} a_{r}+z^{2} a=0, \quad|r| \leq 1, \\
a(1)=\int_{B} a(r) d \nu=\int_{0}^{1} a(r) d \sigma,
\end{gathered}
$$

where $\sigma$ is a probability measure on the Borel subsets of the interval $[0,1)$ defined as

$$
\sigma(E):=\nu(\{r \theta: r \in E, \theta \in S\})
$$

for any Borel subset $E$ of $[0,1)$. Using Fubini's theorem, (4.3) may be rewritten as

$$
0=a(1)-\int_{0}^{1} a(r) d \sigma=\int_{0}^{1} a^{\prime}(r) F(r) d r
$$

where $F(r):=\sigma([0, r))$.

Below $c$ will represent some constant which may vary from equation to equation, and we define $D_{s}:=\frac{1}{s} \frac{d}{d s}$. The smooth solutions of (4.2) are multiples of $a(r):=$ $(r z)^{(2-d) / 2} J_{(d-2) / 2}(r z)$. If $d=2 l+1$, then using (6) on page 46 of [16] we have

$$
a(r)=(r z)^{1 / 2-l} J_{l-1 / 2}(r z)=\left.(-1)^{l} D_{s}^{l}\left(s^{1 / 2} J_{-1 / 2}(s)\right)\right|_{s=r z}=\left.c D_{s}^{l}(\cos s)\right|_{s=r z} .
$$

Now

$$
\frac{d}{d r}(f(r z))=z f^{\prime}(r z)=z\left(\frac{d}{d s} f(s)\right)_{s=r z}=r z^{2}\left(\frac{1}{s} \frac{d}{d s} f(s)\right)_{s=r z}=r z^{2}\left(D_{s} f(s)\right)_{s=r z}
$$

hence $a^{\prime}(r)=c z^{2} r\left(D_{s}^{l+1} \cos s\right)_{s=r z}$. Since $\frac{1}{2} D_{s}$ corresponds to differentiation with respect to $s^{2}$, we have

$$
\left.D_{s}^{l+1}\left(s^{2 j}\right)\right|_{s=r z}=\frac{2^{l+1} j !}{(j-l-1) !}(r z)^{2(j-l-1)}=\frac{1}{r^{2(l+1)}} D_{z}^{l+1}(r z)^{2 j},
$$


so $a^{\prime}(r)=c \frac{z^{(d+2) / 2}}{r^{d}} D_{z}^{(d+1) / 2} \cos (r z)$. Hence, from (4.4), $z$ is a root of

$$
\int_{0}^{1}\left(D_{z}^{(d+1) / 2} \cos (r z)\right) r^{-d} F(r) d r=0
$$

Define

$$
P(z):=\int_{0}^{1} \cos (r z) r^{-d} F(r) d r .
$$

Since $r^{-d} F(r)$ is increasing on $[0,1]$, from the result on page 13 of [15], $P(z)=0$ will have only real roots. Now $P(z)$ is an entire function, and on $|z| \leq R$ we have

$$
|P(z)| \leq \frac{1}{2} \int_{0}^{1}\left(\left|e^{i r z}\right|+\left|e^{-i r z}\right|\right) r^{-d} F(r) d r \leq e^{R} \int_{0}^{1} r^{-d} F(r) d r .
$$

So $P(z)$ is an entire function of order at most 1; see page 16 of [9]. Since the power series of $P(z)$ consists of powers of $z^{2}$, we see that $D_{z}^{k} P(z)$ is an entire function for every positive integer $k$. Further, by writing the expanded form of $D_{z}^{k} P(z)$ as an integral and using arguments used above, one may see that $D_{z}^{k} P(z)$ is of order at most 1. Hence, by Laguerre's theorem $(P(z)$ is real for real $z)$ - see page 96 of [9] we conclude that (4.5) will have only real roots.

We now give a proof of Remark 1.4. Let $d=3$ and $\nu$ be a probability measure concentrated at a point $p$ units from the origin, for some $p \in(0,1)$. Then the associated $\sigma$ is a probability measure on $[0,1]$ concentrated at $p$, and its distribution function is

$$
F(r)= \begin{cases}0, & r \leq p \\ 1, & p<r \leq 1\end{cases}
$$

Then, from (4.5) we need to examine the roots of

$$
\begin{aligned}
0 & =\int_{0}^{1} \frac{D_{z}^{2} \cos (r z)}{r^{3}} F(r) d r=\int_{p}^{1} \frac{\sin (r z)}{r^{2} z^{3}}-\frac{\cos (r z)}{r z^{2}} d r=-\int_{p}^{1} \frac{d}{d r}\left(\frac{\sin (r z)}{r z^{3}}\right) d r \\
& =\frac{\sin p z-p \sin z}{p z^{3}} .
\end{aligned}
$$

If we take $p=1 / 4, w=z / 4$, and note that

$$
4 \sin w-\sin (4 w)=\sin w(1-\cos w)\left(2 \cos ^{2} w+2 \cos w+1\right),
$$

one may see that the equation will have roots which are not real. Note that these roots do not generate the non-zero eigenvalue with the largest real part. The nonzero eigenvalue with the largest real part is actually a Dirichlet eigenvalue associated with a non-radial eigenfunction and is real.

\section{REFERENCES}

1. L. Ahlfors, Complex Analysis, McGraw-Hill, New York (1978). MR.510197 (80c:30001)

2. I. Ben-Ari and R. Pinsky, Spectral analysis of a family of second-order elliptic operators with nonlocal boundary condition indexed by a probability measure, J. Functional Analysis 251(2007), 122-140. MR2353702

3. I. Ben-Ari and R. Pinsky, Ergodic behavior of diffusions with random jumps from the boundary, preprint (2007).

4. K. Burdzy, R. Holyst and P. March, A Fleming-Viot particle representation of the Dirichlet Laplacian, Comm. Math. Phys. 214(2000), 679-703. MR.1800866 (2002c:60130)

5. I. Grigorescu and M. Kang, Brownian motion on the figure eight, Journal of Theoretical Probability 15(2002), 817-844. MR:1922448 (2003f:60144) 
6. I. Grigorescu and M. Kang, Path collapse for an inhomogeneous random walk, J. Theoret. Probab. 16(2003), 147-159. MR1956825 (2004i:60116)

7. I. Grigorescu and M. Kang, Path collapse for multidimensional Brownian motion with rebirth, Statist. Probab. Lett. 70(2004), 199-209. MR2108086 (2005j:60155)

8. I. Grigorescu and M. Kang, Ergodic properties of multidimensional Brownian motion with rebirth, Electron. J. Probab. 12(2007), 1299-1322. MR.2346513

9. A.S.B. Holland, Introduction to the Theory of Entire Functions, Academic Press, New York (1973). MR0447572 (56:5882)

10. E. Kosygina, Brownian flow on a finite interval with jump boundary conditions, Discrete Contin. Dyn. Syst. Ser. B. 6(2006), 867-880. MR2223912 (2007g:60095)

11. William McLean, Strongly Elliptic Systems and Boundary Integral Equations, Cambridge University Press, Cambridge (2000). MR.1742312 (2001a:35051)

12. G. Pólya, Über die Nullstellen gewisser ganzer Funktionen, Math Zeitschrift 2(1918), 352-383. MR 1544326

13. G. Pólya and G. Szegö, Problems and Theorems in Analysis, Volumes One and Two, Translated from the German by C. E. Billigheimer. Reprint of the 1976 English translation. Classics in Mathematics. Springer-Verlag, Berlin (1998). MR1492448

14. E. Stein and G. Weiss, Introduction to Fourier Analysis on Euclidean Spaces, Princeton University Press (1971). MR.0304972 (46:4102)

15. G. Szegö, Inequalities for the zeros of Legendre polynomials and related functions, Transactions of the AMS 39(1936), 1-17. MR.1501831

16. G.N. Watson, A Treatise on the Theory of Bessel Functions, Cambridge University Press, Cambridge Mathematical Library Edition (1995). MR1349110 (96i:33010)

Department of Mathematical Sciences, University of Delaware, Newark, Delaware 19716

E-mail address: yleung@math.udel.edu

Department of Mathematical Sciences, University of Delaware, Newark, Delaware 19716

E-mail address: wli@math.udel.edu

Department of Mathematical Sciences, University of Delaware, Newark, Delaware 19716

E-mail address: rakesh@math.udel.edu 